\title{
High Spin States in Odd-A Gadolinium Isotopes
}

\author{
S. Mohammadi", H. Aghahasani \\ Department of Physics, Payame Noor University, PO BOX 19395-3697 Tehran, Iran \\ *Corresponding Author: smohammadi1958@yahoo.co.uk
}

Copyright (C) 2014 Horizon Research Publishing All rights reserved.

\begin{abstract}
We have developed a special computing code for calculation of nuclear shape changes and quadrupole moments ( Q) of Gadolinium Isotopes. It has been shown from these calculations that by increasing neutron number, deformation parameter also increase for more heavier isotopes which means more deformation from spherical shape. By comparison with Nilsson level diagrams we can infer quadrupole deformation parameter $\left(\beta_{2}\right)$ and calculate quadrupole moments of these isotopes.
\end{abstract}

Keywords Yrast States, Backbending, Deformation Parameter, Quadrupole Moment, Shape Change

\section{Introduction}

We know that nuclei in many cases have large quadrupole moments (Q) and they don't behave like a point charge, rather a spherical or elliptical shape with an axis of symmetry is considered for these nuclei. By knowing the quadrupole moments, we can measure deformation parameters which can be used to define the shape of nuclei. There are different theoretical and experimental methods for calculation and measurement of nuclear electric quadrupole moments [1-7].

The effect of backbending has been observed experimentally in the ground state rotational band of some deformed nuclei. The effect occurs due to the rapid increase of the moment of inertia with rotational frequency towards the rigid value.

When the rotational energy exceeds the energy needed to break a pair of nucleon, the unpaired nucleon goes into different orbits, which result in change of the moment of inertia. An explanation of this effect is due to a disappearance of the pairing

correlation by the action of Coriols forces, where the nucleus then undergoes a phase transition from a super fluid state to a state of independent particle motion.

Other proposed explanations such as rotational alignment and centrifugal stretching, along with the former, could be described in terms of band crossing;

the case where the breakup of one pair of nucleons providing a large angular momentum, which may couple with the collective rotation to produce a new band.
This effect makes up the backbending phenomena, which is experimentally observed in $I-\omega^{2}$ plot as a radical change in the behavior of the angular momentum from a linearity to a stretching and then back to linear again.

In this paper we present a new method for calculation of quadrupole moments of odd-A Gadolinium isotopes for the first time. By study of rotational gamma-decay cascades in different bands of these isotopes and drawing the experimental yrast level energies versus moments of inertia for each band, we look for Backbending phenomenon [8]for each Ir isotope. If there is a Backbending, then it means that there is a change of moment of inertia, which is happening by excitation of a nucleon to another state with different angular momentum. Thus changing the total spin of nucleus. By comparison with related Nilsson diagram [9], we can find the location of displaced nucleon and thus find the related quadrupole deformation parameter $\left(\beta_{2}\right)$ at that excitation energy. By finding the deformation parameter we can calculate the quadrupole moment of the deformed isotope and study shape changes.

\section{Theoretical Calculations and Discussion}

Nuclei can be obtained in very high angular momentum states, mainly through heavy-ion induced reactions (HI, xn). The states that are populated subsequently, decay, through a series of statistical low-spin transitions, into the high-spin lower energies yrast structure .

It has been shown that a large amount of angular momenta can be obtained by collective motion (i.e. a coherent contribution of many nucleons to the rotational motion). It is important that the nucleus exhibits a stable, deformed shape. Subsequently, rigid rotation will contribute angular momentum $\mathrm{J}$ and energy $\mathrm{E}$ according to the expression

$$
E=\frac{\hbar^{2}}{2 I} J(J+1)
$$

Where I is the moment of inertia.

Besides th collective rotational motion, angular momentum can be acquired by non-collective motion. Here, the alignment of the individual nuclear orbits along the nuclear symmetry axis contributes to the total nuclear spin. The system does not have large deformed shapes but remains 
basically spherical or weakly deformed.

The excited states should cascade down toward the ground state through a sequence of E2 gamma transitions. The observation of these cascade $\mathrm{E} 2$ transitions provides a way to study these excited states. In particular, we can study whether the assumption of a fixed constant moment of inertia remains valid at such high excitations. One way to test this assumption is to plot the energies of the states against $\mathrm{I}(\mathrm{I}+1)$ and to see if the slope remains constant. Figure 1 is an example of such a plot for ${ }^{158} \mathrm{Er}$ and ${ }^{174} \mathrm{Hf}$ nuclei and as it can be seen there appears to be some deviation from the expected linear behavior[8].

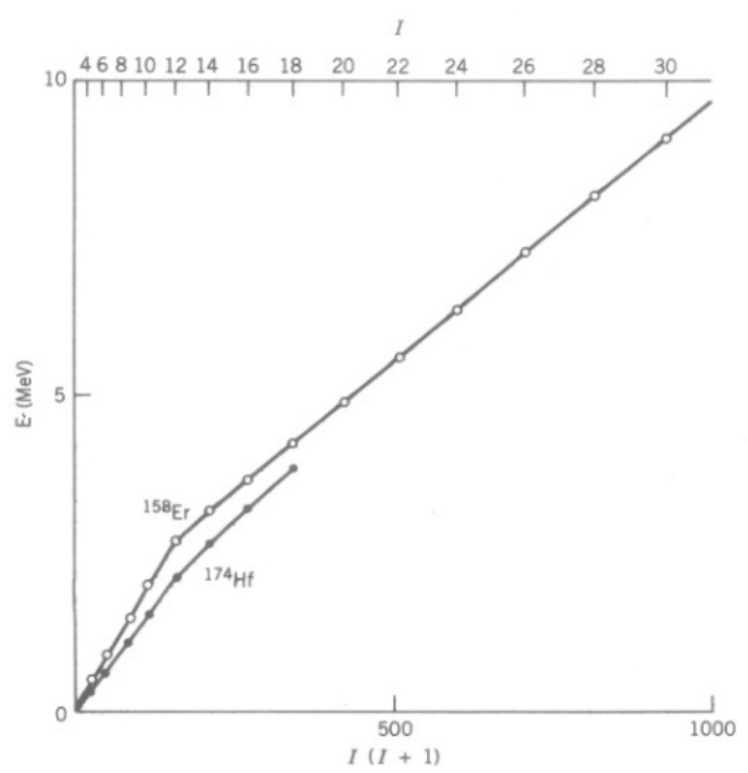

Figure 1. E versus $\mathrm{I}(\mathrm{I}+1)$ plot for Er and Hf nuclei[8].

If we assume that the moment of inertia is not constant but increases gradually as we go to more rapidly rotating states. This effect known classically as "centrifugal stretching" would not occur for a rigid rotor but would occur for a fluid. Because rotating nuclei have moments of inertia somewhere between that of a rigid rotor and of a fluid, it is not surprising that centrifugal stretching occurs. There is a more instructive way to plot the data on the rotational structure. From equation (1) the energy of a transition from state I to the next lower state I -2 is

$$
E(I)-E(I-2)=\frac{\hbar^{2}}{2 g}(4 I-2)
$$

Or by rearranging the terms

$$
\frac{2 g}{\hbar^{2}}=\frac{4 I-2}{E(I)-E(I-2)}
$$

By plotting the left hand side of the above equation versus the square of rotational frequency $\omega^{2}$, there appears to be a gradual increase in moment of inertia among the lower angular momentum states, then a radical change in behavior and then again a return to the gradual stretching as shown in figure 2. This effect which is known as Backbending occurs in some heavy nuclei because the rotational energy exceeds the energy needed to break a pair of coupled nucleons. When this effect occurs, the unpaired nucleons go into different orbits and change the nuclear moment of inertia [8].

During the last decade nuclear high spin states have been the subject of experimental and theoretical studies. The fascinating progress in this new field has been made possible by the essential development in experimental on exciting and detecting the high spin states in nuclei. A part from such interesting expectations as the existence of a super-back-bending or super-deformation, the research in this new field can also be considered as providing a tool for testing the validity of nuclear models under extreme conditions. As an example, the back-bending phenomena discovered in 1970 [10] is now interpreted as being due to the crossing of the ground state band with an aligned quasi-particle band .

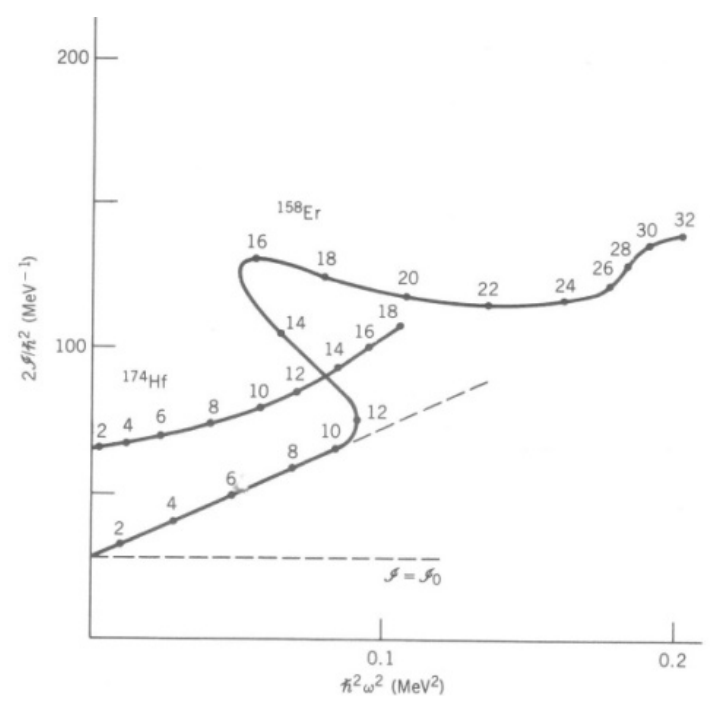

Figure 2. Moment of inertia versus $\omega^{2}$ showing Backbending [8].

\section{Shape Changes in Gadolinium Isotopes}

When looking at the nucleus of an atom it is considered, in general, to be spherical in shape. However, in reality, this is not the case; the nucleus will have a deformation from this spherical shape depending on the structure of the nucleus and the internal forces produced by this structure. Over the years, different theoretical models have been created to try to calculate these deformations, to predict the shape of nuclei. However, the accuracy of these models always vary due to factors that had not been considered or situations occurring where the model predicts very well in certain regions compared to other models, but not as well for others regions.

Gadolinium Isotopes have 64 protons. In this paper we studied rotational gamma decays of Isotopes from $\mathrm{A}=139$ to $A=153$. Figures 3 and 4 clearly show Backbending for $A=139,143$ isotopes. Using this plot and comparison with Nilsson diagram for neutrons with $\mathrm{N}=75$, we find quadrupole deformation parameter for this isotope $\beta_{2}=$ 
0.263. From this finding and using the Grodzins formula for quadrupole moment [11],

$$
\mathrm{Q} \cong \frac{3}{\sqrt{5 \pi}} \mathrm{Zr}_{0}{ }^{2} \mathrm{~A}^{\frac{2}{3}} \beta_{2}
$$

This equation is a simplification of the far more expanded original version, but this will fit the requirements for this project as the later terms make exceptionally small differences to the results and would only need considering in cases where the utmost accurate value of a nuclei's quadrupole moment is required. Even though this report is dealing with how accurate this theoretical method is, these extra units in the calculation would make minimal difference in the comparison and so would have little effect on the overall review of its accuracy.

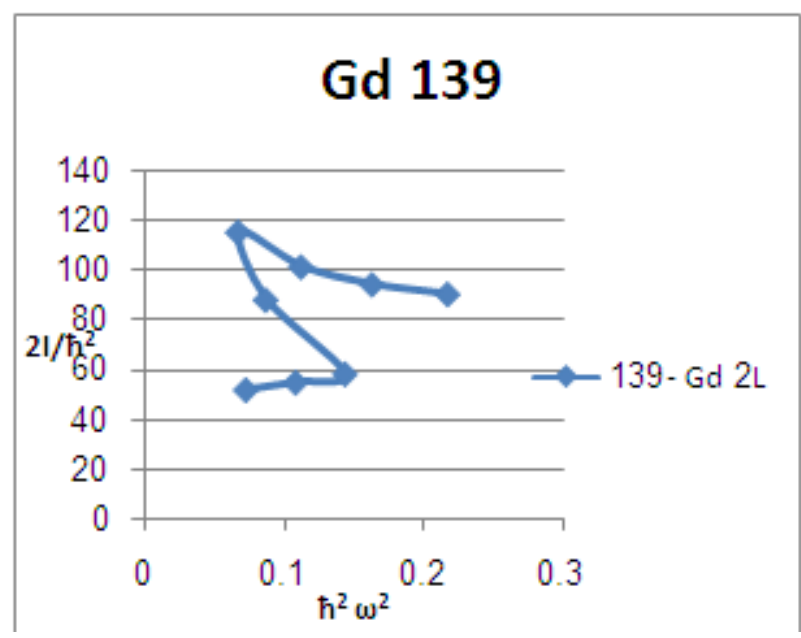

Figure 3. Backbending for $\mathrm{A}=139$ Gadolinium isotope.

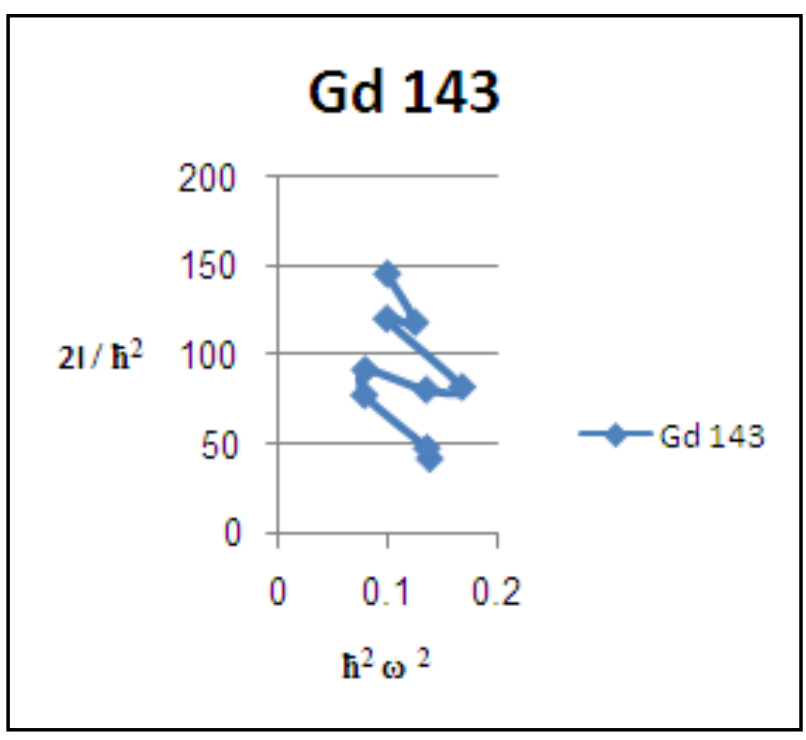

Figure 4. Backbending for $A=143$ Gadolinium isotope

The ground state and excited state of Gadolinium isotopes are presented in table 1 .We can calculate the quadrupole moment, which is a measure of deformation of nucleus from spherical shape. The changes in deformations and calculated quadrupole moments for odd-A Gadolinium isotopes are summarized in table 2.

The parameters $\varepsilon$ and $\beta$ are related to each other by the following relations:

$$
\begin{aligned}
\varepsilon_{2} & =0.95 \beta_{2} \\
\varepsilon_{4} & =\varepsilon_{2}^{2}{ }_{2} / 6 \\
\varepsilon_{4} & =0.95 \beta_{4}
\end{aligned}
$$

By having accurate values of the intrinsic quadrupole moment, due to the experimental data, it is possible to compare the theoretical values for $Q$ for all nuclei, and for when it is used as its calculated value for each nuclei. This way it is possible to see how closely the theoretical values can predict the true $Q$ value, as well as seeing how much of an improvement using $\beta_{4}$ in the equations makes to its prediction. Although $\beta_{2}$ is the main deformation parameter to affect the nuclei's shape, by analyzing the difference that occurs due to leaving $\beta_{4}$ in or out of the equation will give an idea as to how much of an effect $\beta_{4}$ has on the nuclei.

Table 1. The ground state and excited state of Gadolinium isotopes

\begin{tabular}{|c|c|c|}
\hline Isotope & Ground State & $\begin{array}{c}\text { Excited } \\
\text { State }\end{array}$ \\
\hline${ }^{139}{ }_{64} \mathrm{Gd}$ & $9 / 2^{-}[514]$ & $11^{-}[505]$ \\
\hline${ }^{141}{ }_{64} \mathrm{Gd}$ & $1 / 2^{+}[400]$ & $3 / 2^{+}[512]$ \\
& $1 / 2^{+}[411]$ & \\
\hline${ }^{143}{ }_{64} \mathrm{Gd}$ & $1 / 2^{+}[400]$ & $3 / 2^{+}[402]$ \\
\hline${ }^{145} \mathrm{Gd}$ & & $13 / 2^{+}[606]$ \\
\hline${ }_{64}^{147} \mathrm{Gd}$ & $7 / 2^{-}[514]$ & $5 / 2^{-}[523]$ \\
\hline${ }^{149}{ }_{64} \mathrm{Gd}$ & $7 / 2^{-}[514]$ & \\
\hline
\end{tabular}

Table 2. Deformation parameters and Quadrupole moments for Gadolinium isotopes

\begin{tabular}{|c|c|c|c|c|c|}
\hline Isotope & $\varepsilon_{2}$ & $\beta_{2}$ & $\varepsilon_{4}$ & $\beta_{4}$ & $\mathrm{Q}$ \\
\hline${ }_{64}^{139} \mathrm{Gd}$ & 0.23 & 0.263 & 0.0104 & 0.0109 & 503.228 \\
\hline${ }^{141}{ }_{64} \mathrm{Gd}$ & 0.27 & 0.284 & 0.0121 & 0.0127 & 507.668 \\
\hline${ }^{151}{ }_{64} \mathrm{Gd}$ & 0.43 & 0.452 & 0.0301 & 0.0315 & 1110.31 \\
\hline${ }_{64}^{153} \mathrm{Gd}$ & 0.1 & 0.105 & 0.0016 & 0.0017 & 233.42 \\
\hline
\end{tabular}

As it can be seen from the above table, with increasing neutron numbers in Gadolinium isotopes, changes in shape occur. These smooth changes are all occur in prolate shapes.

This is due to the shape of the nuclear structure being drawn to an oblate shape as $\beta_{2}$ is negative, where as in most cases $\beta_{2}$ is positive, implying a prolate nucleus shape for the nuclei. 
The meaning of this change to the nuclei's physical shape is depicted in figure 5 below with prolate being rugby ball shaped, where as oblate is a disk shape.

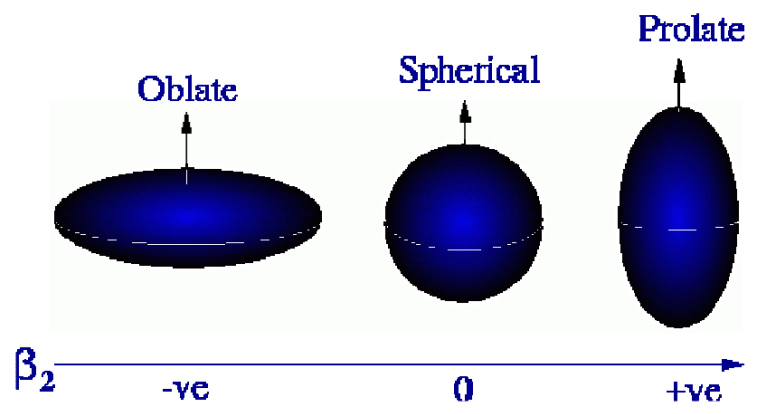

Figure 5. Picture of how the shape of the nucleus changes with $\beta_{2}$ from negative to positive.

\section{Conclusions}

The present work suggests that the Variable Moment of Inertia with is a successful tool in studying ground state energy levels in deformed Gadolinium nuclei up to high spin states. Predictions of the model give fairly accurate description of the backbending phenomena in these isotopes.

The appearance of backbending can be attributed due to the smallness of critical rotational frequency of the protons than for neutrons, therefore, the behavior of the proton pair at high spins seems to be decisive for such effect. On the other hand, the absence of backbending in some isotopes may be ascribed to the presence of stable octupole deformation in them.

It has been shown from these calculations that by increasing neutron number of Gadolinium isotopes, deformation parameter also changes which means more shape changes from spherical shape. By comparison with Nilsson level diagrams we can infer deformation parameter $\left(\beta_{2}\right)$ and calculate quadrupole moments of these isotopes.
This means that there are shape changes which are mostly prolate deformations in these isotopes.

\section{REFERENCES}

[1] A. DeShalet and H. Feshbach, Theoretical Nuclear Physics, Volume 1 John Wiley \& Sons, 1990.

[2] A.M. Mårtensson-Pendrill, et al, Isotope shifts and nuclear-charge radii in singly ionized $40-48 \mathrm{Ca}$, Phys. Rev. A 45, 1992, pp4675-4681

[3] T. Otsuka, N. Fukunishi and H. Sagawa, Structure of exotic neutron-rich nuclei, Phys. Rev. Lett. 70, 1993, pp1385-1388

[4] T. Baumann, et al, Longitudinal momentum distributions of 16,18C fragments after one-neutron removal from 17,19C, Phys. Lett. B439, 1998, pp256-261

[5] B.A. Brown and B.H. Wildenthal, Empirically optimum M1 operator for sd-shell nuclei, Nucl. Phys A, 474, 1987, pp290-306

[6] J. Cederberg, et al, Nuclear electric quadrupole moment of 6Li, Phys. Rev. A57, 1998, pp 2539-2543

[7] W. Nörtershäuser, et al, Isotope shifts and hyperfine structure in the $3 \mathrm{~d} 2 \mathrm{DJ} \rightarrow 4 \mathrm{p} 2 \mathrm{PJ}$ transitions in Calcium II, Eur. Phys. J. D 2, 1998, pp33-39

[8] K.S. Krane, 1988, Introductory Nuclear Physics, John Wiley \& Sons, ISBN 0471859141

[9] S.G. Nilsson, Binding States of Individual Nucleons in Strongly Deformation Nuclei, Das-Math. Pys., Vol.29, No.16, 1955.

[10] A. Johnosn, H. Ryde, J. Sztorkier, Phys. Lett. B 34 605, 1971.

[11] L. Grodzins , Phys.Lett.2, 88, 1962. 